\title{
The COSPAR Capacity Building Initiative
}

\author{
Carlos Gabriel \\ COSPAR Panel for Capacity Building (PCB) \\ COmmittee for SPace Research \\ Heidelberg, Germany \\ juan.carlos.gabriel@gmail.com \\ Diego Altamirano \\ SPA, University of Southampton / COSPAR PCB \\ Southampton, United Kingdom \\ Dieter Bilitza \\ George Mason University / COSPAR PCB \\ Washington, USA \\ Raffaella D'Amicis \\ IAPS/INAF / COSPAR PCB \\ Rome, Italy \\ Jean-Louis Fellous \\ COSPAR Secretariat \\ Paris, France
}

\begin{abstract}
The Capacity Building Programme (CBP) is considered today one of the flagships of COSPAR (Committee on Space Research) activities. The programme started in 2001 as a tentative project designed to widen expertise in space sciences and promote the use of data archives from space missions in developing countries, as a way to foster in those regions of the world high quality scientific activities. In the past 19 years a total of 35 COSPAR workshops have been held, involving more than 1000 advanced students and young researchers in 21 different developing countries. Participants have learnt in a highly practical manner how to analyse data from diverse space missions, covering practically all Space Science disciplines, from Astronomy to Earth Observation, from Solar Physics to Planetary Sciences, including Ionosphere, Magnetospheric sciences and even Planetary Crystallography. A key to the success of the CBP has been the strong and selfless engagement of internationally high ranked scientists as well as of the space agencies, ESA, NASA and JAXA. I will discuss in this presentation the history and current status of the Programme, but emphasise the changes we are introducing to make it better, more efficient and wider in scope.
\end{abstract}

Keywords-Space Sciences; Capacity Building; Space Educational Activities; COSPAR

\author{
Alexi Glover \\ ESA/ESOC / COSPAR PCB \\ Darmstadt, Germany \\ Aaron Janofsky \\ COSPAR Secretariat \\ Paris, France \\ Ernesto López-Baeza \\ Universidad de Valencia / COSPAR PCB \\ Valencia, Spain \\ Mariano Méndez \\ KAI, University of Groningen / COSPAR PCB \\ Groningen, the Netherlands \\ Randall Smith
CfA / COSPAR PCB
Cambridge, USA \\ Z. Bob Su \\ University of Twente \\ Twente, the Netherlands
}

\section{INTRODUCTION}

The Committee on Space Research (COSPAR) gathers national and international science academies and institutions of 46 countries, contributing to promote space sciences activities.

The Capacity Building Programme (CBP) was initiated by a COSPAR Associate, Professor Peter Willmore (Univ. of Birmingham) at the beginning of the millennium with the following purposes:

- to increment the knowledge and usage of space data public archives together with corresponding tools in developing countries. This should extend the research horizons in those countries and make sure that local scientists are aware of the opportunities brought by those data and tools, publicly available,

- to instruct practically the students in the practical usage of archives and associated software,

- to promote professional bonds between the CB workshop participants and experienced scientists acting as teachers and supervisors, reducing the inherent isolation suffered by scientists in developing countries. 
With these purposes a program of highly practical scientific regional workshops was conceived, with a duration of approximately two weeks, which are based on space science missions offering free access to their data and reduction and analysis tools. A demonstrated interest in the scientific area in the region where the workshops take place is one of the prerequisites, since the basic idea is that the workshop participants make use of the gained knowledge in their later scientific career as a contribution to that country / region.

\section{BASIC (SPACE) SCIENCES IN DEVELOPING}

\section{COUNTRIES}

There is a strong correlation between scientific productivity and present and future economic development of a country. There are even indicators that countries focusing their scientific activity in applied sectors grow slower than those doing it in basic areas [1]. It is also common sense that applied sciences research needs a basic sciences substrate: "There is no applied science without a systematic set of 'pure' scientific knowledge behind, and almost all 'pure' sciences are continuously applied for solving concrete problems" [2].

Beyond these considerations we value the basic concept that science knowledge shouldn't have any ownership, but has to be a common good of human kind, distributed in all directions in this uneven world.

Several factors inhibit development of basic sciences in developing countries. Fundamental space sciences particularly suffer due to high cost of experiments. Mitigating this difficulty is the fact that we live in the era of information distribution in which all kinds of data and knowledge are freely offered. The development of open source code and tools offered through web services give a new dimension to the accessibility of science data. Particularly in astrophysics, the concept of public observatory, developed in the 20th century, has favored the development of a culture of open access to science data. As of today, any large astrophysical space mission is expected to eventually disseminate not only its results but all of its scientific data in open public archives. The archives have become a fundamental source of scientific research. The highest point in this development has been the initiative of the Virtual Observatory (VO) [3], which interconnects all the main astrophysical archives (both from space and ground observatories), after a rapid process of standardisation has taken place. This is maximising usage of the archives, facilitating user access to data and visualisation and analysis tools.

This example of openness and active scientific data dissemination is followed more and more in other disciplines. Planetary and solar physics data for instance have profited from sharing resources with astrophysics in the archive development sector of the large space agencies.

\section{ARCHIVES AND ANALYSIS TOOLS}

A large number of modern data archives do exist today, in most cases every single one associated to a space mission, an experiment, or a telescope. The European Space Agency (ESA) develops and maintains them in its European Space Astronomy Centre (ESAC) in Spain. Their suite of archives comprises data from astronomy, planetary and solar missions of most diverse types [4]. Enormous amounts of data are presented in different stages of reduction, from raw to fully processed, going far beyond a simple offer of data collections. Much effort is put into helping and boosting the possible exploitation of all the data contained in the archives, with the aim of maximising scientific return, i.e., to reach the largest possible number of users, independently of nationality or professional affiliation.

While the archives generally offer calibrated and processed data to different levels with a standard reduction, the maximal extraction of the science contained is often only possible through the usage of interactive data analysis tools, capable of optimising the signal to noise ratio according to the specific scientific purpose. Most large projects offer these kinds of analysis tools publicly with the same aim as the one mentioned for the archives: maximisation of scientific return [5].

The COSPAR Capacity Building workshops have been structured around these two elements: public archives and freely available analysis tools. The first workshop of the Programme was held in 2001 at the National Institute of Space Research (INPE) in Sao José dos Campos, Brazil, devoted to X-ray Astrophysics, and based on the space missions Chandra (NASA) and XMM-Newton (ESA). Both missions were already providing in 2001 advanced scientific archives with a reasonable amount of public data and data analysis software freely distributeda. This workshop has been organised eight times in several countries (Brazil, India, South-Africa, Egypt, Argentina, China and Mexico), representing more than $20 \%$ of all CB workshops.

While publicly accessible resources make it possible to perform scientific research at a high level at any location in the world, users come mainly from developed countries for several reasons. The two most important causes are the lack of knowledge about the existence of publicly accessible resources in developing countries, and a clear deficit in developing regions in space sciences, since related disciplines do not belong to the traditional local research areas.

A clear impulse for profiting scientifically in developing countries from those resources could be given, therefore, through an educational program, trying to revert this situation. So the Capacity Building Programme was born, sustained by COSPAR [6], with the main aim of encouraging scientists from developing regions to make use of scientific space data and tools.

${ }^{\text {a} E i g h t e e n ~ y e a r s ~ l a t e r ~ b o t h ~ m i s s i o n s ~ a r e ~ a l i v e ~ a n d ~ e x t r e m e l y ~ p r o d u c t i v e . ~ A ~ l o t ~ m o r e ~ d a t a ~ t h a n ~ i n ~ t h o s e ~ d a y s ~ a r e ~ a v a i l a b l e ! ~}$ 


\section{BROADENING OF THE PROGRAMME}

After the first two workshops in X-ray astronomy, the CBP started to broaden its range, until today most of, if not all, the space science disciplines are covered.

In the last eighteen years a total of 35 workshops have taken place, covering all areas of astronomy (gamma, Xray, infrared, optical and ultraviolet), along with planetary sciences, magnetospheric physics, Sun-Earth interactions, diverse Earth observation fields, space weather and even crystallography in space. They have taken place in 21 different developing countries. The initial frequency of an annual workshop has been tripled and more than 1000 students from developing countries have participated so far.

\section{LEARNING NEEDS AND CHARACTERISTICS OF THE CBP WORKSHOPS}

The typical target of the workshops is a young scientist, $\mathrm{PhD}$ or Master student, or recently finished with his/her studies. The learning needs start often with the specific type of science in areas not mastered by the participants. These may simply be not studied by existing researchers in the region, or lie in a different part of the electromagnetic spectrum to those they have been exposed to. The instruments on board space missions are generally less known or totally unknown, as is often true with their data characteristics. Also, the usage of science archives as fundamental source for research is often a novelty. In many cases, the analysis techniques and obviously the mission specific analysis tools, as key for the future work, are the central elements of the practical work during the workshops. Of course, all these elements depend on the specificities of the area, which can be highly different, as it can be assumed from the broadness of the Programme. In the general case a workshop's agenda includes lectures on all the topics mentioned above, but the central element of it is the individual project of the student, who will use the new knowledge and techniques learnt to extract science from the data (s)he is going to work with, be this alone or in teams. All these elements together define a general duration of a workshop of two weeks, as a compromise between the learning necessities and the real possibilities to go through a small scientific project under the active supervision of the international experts, who are at the same time lecturers of the workshop.

The average $\mathrm{CB}$ workshop has $25-35$ participants. Around 10 lecturers / project supervisors are needed to cover the diverse topics and to ensure active and effective supervision. Participants are recruited internationally by scientists and data analysis specialists in the area of the workshop. The projects can be carried out individually or in teams; appropriate supervisors are chosen and correspond to the specific project. Each projecthas one or two supervisors. The workshops end with an individual presentation by every student (oral talk or poster) in which the comprehension of the methodology and the ability to work with data and specific area tools have to be demonstrated.
One fundamental element in the COSPAR CBP philosophy is that students and lecturers reside in the same hotel during the entire duration of the workshop, sharing also all meals. The direct contact with international specialists is of paramount importance for students from developing countries in their scientific careers.

\section{THE CBP ASSOCIATED FELLOWSHIP}

In 2010 we complemented the Programme with an associated fellowship. Fellowships are open to young scientists who have been participants at one of the COSPAR Capacity Building workshops. The objective is to enable participants to build on skills gained during the workshops. Fellowships consist of visits of 2-4 weeks duration for the purpose of carrying out joint research in laboratories that collaborate with COSPAR in organizing the fellowship program.

\section{NUMBERS AND RESULTS}

During the 19 years of the CBP, COSPAR has held 35 workshops in almost all space science disciplines, as specified above. The workshops have taken place in 21 developing countries and more than 1000 students have participated in them. In 2019 we are organizing another two workshops and adding also two additional host countries to our list.

Concerning the associated fellowships, their number has been very stable since the start of the programme in 2010. A total of 51 visits have taken place, most of them in Europe and USA, with several publications of results as the outcome. Practically all fellowships have served to enhance and expand the knowledge acquired in a new research area.

The validity of the CBP pedagogical concepts is shown in a very steep learning curve of most students during the workshops as well as in a very efficient knowledge transfer. The deep relationship established between international experts and students during the two weeks of each workshop is fundamental for helping the latter in their careers. The importance of the relationship is also proven in the many cases of research visits by former workshop participants to leading institutes throughout the world, in the framework of an CBP associated fellowship but not limited to it.

The students' satisfaction level with respect to the workshops is very high, as measured through questionnaires. The evaluation question related to "significant benefit in attending the workshop" is answered almost unanimously with maximum satisfaction.

\section{THE FUTURE}

While the results are so far very satisfactory, and COSPAR plans to continue with the CBP along the same lines, we have decided to take action on three points further to discussion during the COSPAR Scientific Assembly in 2018: 
- Necessity of understanding better the efficiency of the CB workshops. While we have qualitative answers showing the usefulness of the Programme, we do not have quantitative results in terms of relating a workshop to the later career of participants. As an answer to this we have created an Alumni structure with one member of the PCB acting as its Chair. Every workshop held from this year on is expected to create an individual cell of this structure, self-organised by its participants, with one of the students acting as Alumni Delegate. The aim is to organise a yearly event per cell, i.e., a videoconference, by which the former participants of a single workshop can interact and give feedback about their careers, especially as they relate to their workshop participation. We expect a high level of involvement of former students in these events. It should help them maintain a close contact to COSPAR and to colleagues in the field. It could also be a source for recruiting young people as lecturers or data analysis specialists for future workshops. We have many individual examples of former workshop participants helping to organise a later workshop (one member of the renewed Panel on Capacity Building (PCB) was even a student at our first workshop), but the Alumni structure is especially suited for helping with this aspect. Understanding the individual careers after a workshop will also allow us to look more closely into diversity questions, going beyond the level we can ensure in an individual workshop itself (as we aim through gender quotes, geographical distribution, etc.)

- Homogenisation of the different areas in the CBPis also seen as a necessity. In order to introduce greater interaction between the responsible members of the different disciplines, we have started to hold bimonthly PCB videoconferences. Written procedures for preparation, execution as well as financial control of a workshop are in the process of being drafted as a result of the first meeting. These procedures should help ensure that all areas are treated equally, and also that the principles on which the COSPAR CB Programme is based are well understood, shared and executed by all members of the PCB at each organized workshop. Additionally, homogenisation will secure acceptable ratios of local to COSPAR funding, and of local to regional participants, but also gender diversity where it is needed. An important measure to homogenise the diverse areas is the introduction of biannual calls for workshop proposals. In March and October the PCB decides on workshops to take place between 9 and 20 months later.

- Reflection about the possibility of creating a new type of workshop built around very small satellites (i.e. Cubesats, or Nanosats). Multi-disciplinary work could be fostered at these workshops which would be aimed at younger students than those participating in the typical COSPAR CB workshop (i.e. pre-graduates, even high school students). A high level of team building would be one of the distinct characteristics of such a workshop, stretching through the different disciplines necessary for a practical project around a small satellite. Given the level of COSPAR finances, and to avoid jeopardising the traditional $\mathrm{CB}$ Programme, a different funding model would need to be pursued, in which large institutions (UNESCO, EU) but also big private sponsors could contribute. Such workshops could represent COSPAR's educational answer to the new era of small satellites, aiming to help the developing world to avoid missing out on the opportunities provided by these new technologies. The planned COSPAR symposium and CB workshop on small satellites in November 2019 are a perfect platform for giving such ideas concrete form by discussing them with specialists in this area.

\section{SUMMARY}

The CBP is seen today as one of the flagship activities of COSPAR. After almost 20 years of activity we can look back with pride on the 35 capacity building workshops with more than 1,000 students in developing countries which have contributed significantly to the space scientific activities in the regions in which they have been held. While we plan to continue with a similar pace of activity in the coming years we are introducing changes which should make the Programme better and wider in scope:

- We have created an Alumni organization to attract younger scientists and encourage them to maintain a durable relationship to COSPAR;

$\circ \mathrm{We}$ have introduced half-yearly calls for workshop proposals to ensure fairness vis-a-vis the different disciplines, as well as streamlining procedures for organizing and conducting the workshops;

- We are evaluating the feasibility for a new workshop type of multidisciplinary nature, devoted to small satellites and aimed at younger students.

\section{REFERENCES}

[1] Jaffe K., et al., 2013, PLoS ONE, 8, 66239

[2] Sabino C., 1992, "El proceso de investigación"

[3] Hanisch R.J. 2006, in Gabriel C., Arviset C., Ponz D., Solano E., eds, Astronomical Data Analysis Software and Systems XV Vol. 351 of Astronomical Sociaty of the Pacific Conference Series, The Virtual Observatory in Transition. p. 765

[4] Arviset C., et al., 2014, in Manset N., Forshay P., eds, Astronomical Data Analysis, Software and Systems XXIII Vol. 485 of Astronomical Society of the Pacific Conference Series, Long-Term Strategy for ESA Science Archives. P. 159.

[5] Gabriel C., et al., 2004, in Ochsenbein F., Allen M. G., Egret D., eds, Astronomical Data Analysis Software and Systems (ADASS) XIII Vol. 314 of Astronomical Society of the Pacific Conference Series, The XMM-Newton SAS Distributed Development and Maintenance of a Large Science Analysis System: A Critical Analysis. p. 759

[6] Willmore P., 2002, "Widening Expertise in Space Science in Developing Countries". p. 20 